\title{
Efeito de frações inspiradas de oxigênio e modalidades ventilatórias diferentes sobre a idade de cães
}

\author{
[Effects of oxygeninspired fractions and different ventilatory modalities on \\ the age of dogs] \\ K.S. Fuchs, L. Rosa, R. Tochetto, F. Comassetto, C.D.L. Cancellier, \\ S.J. Ronchi, M.G. Luciane, N. Oleskovicz
}

Centro de Ciências Agroveterinárias - Universidade do Estado de Santa Catarina - Florianópolis - SC

\begin{abstract}
RESUMO
Objetivou-se avaliar diferentes modalidades ventilatórias em cães de diferentes idades submetidos à fração inspirada de oxigênio $\left(\mathrm{FiO}_{2}\right)$ de $40 \%$ e 100\%. Foram utilizados 36 cães de três grupos etários (GJ: 0-5; GA: 5-10 e GG: 10-15 anos), sem padronização de peso, sexo, raça e procedimento cirúrgico. Foram prémedicados com acepromazina e morfina $(0,02 \mathrm{e} 0,5 \mathrm{mg} / \mathrm{kg})$, induzidos à anestesia geral com propofol doseefeito, manutenção do plano anestésico com isoflurano em $1,3 \mathrm{~V} \%$ e fornecimento de oxigênio conforme a $\mathrm{FiO}_{2}$ estabelecida para o grupo. Os animais foram submetidos a quatro diferentes modalidades ventilatórias: ventilação espontânea (VE), ventilação ciclada a volume (VCV), ventilação ciclada a pressão (VCP) e ventilação ciclada a pressão com PEEP (VCPP), e permaneceram 30 minutos em cada modalidade. Os parâmetros cardiovasculares mantiveram-se estáveis para todas as $\mathrm{FiO}_{2}$, modalidades ventilatórias e idades. Com relação aos parâmetros ventilatórios, na $\mathrm{FiO}_{2} 100 \%$, foram observados $\mathrm{PaCO}_{2}$ de $45 \mathrm{mmHge}$ e $29 \%$ de shunt, enquanto a $\mathrm{FiO}_{2} 40 \%$ apresentou $\mathrm{PaCO}_{2}$ de $43 \mathrm{mmHg}$ e $13 \%$ de shunt. Em relação às diferentes idades, os animais adultos e geriátricos apresentaram maiores valores de shunt $(26 \%$ e $22 \%)$ e $\mathrm{PaCO}_{2}$ $(44 \mathrm{~mm} / \mathrm{Hg}$ e $46 \mathrm{~mm} / \mathrm{Hg})$. Conclui-se que a fração inspirada de $40 \%$ e a modalidade ventilatória ciclada a volume mostraram-se mais eficientes.
\end{abstract}

Palavras-chave: cães, modalidades ventilatórias, fração inspirada de oxigênio, idade, hemogasometria

\begin{abstract}
The objective was to evaluate different ventilatory modalities in dogs of different ages submitted to the inspired fraction of oxygen $\left(\mathrm{FiO}_{2}\right)$ of $40 \%$ and $100 \%$. Thirty-six dogs from three age groups (GJ 0-5, GA 5-10 and GG 10-15 years) were used, without standardization of weight, gender, race and surgical procedure. They were premedicated with acepromazine and morphine $(0.02$ and $0.5 \mathrm{mg} / \mathrm{kg})$, induced to general anesthesia with propofol doseleffect, maintenance of the anesthetic plane with isoflurane in $1.3 \mathrm{~V} \%$ and oxygen supply according to $\mathrm{FiO}_{2}$ established for the group. The animals were submitted to 4 different ventilation modalities, spontaneous ventilation (VS), volume-cycled ventilation (VCV), pressure-cycled ventilation (VCP) and pressure-cycled ventilation with PEEP (VCPP) and remained 30 minutes in each modality. The cardiovascular parameters remained stable for all $\mathrm{FiO}_{2}$, ventilatory modalities and ages. Regarding ventilatory parameters, in $\mathrm{FiO}_{2}$ and $\mathrm{PaCO}_{2}$ of $45 \mathrm{mmHg}$ and 29\% of shunt, in $\mathrm{FiO} 2 \mathrm{100 \%}$, $\mathrm{PaCO}_{2}$ of $43 \mathrm{mmHg}$ and $13 \%$ of shunt were observed. Regarding the different ages, adult and geriatric animals presented higher values of Shunt (26 and 22\%) and $\mathrm{PaCO}_{2}(44$ and $46 \mathrm{mmHg}$ ). It was concluded that the inspired fraction of $40 \%$ and the volume-cycled ventilatory modality were more efficient.
\end{abstract}

Keywords: dogs, ventilatory modalities, oxygen inspired fraction, age, hemogasometry

Recebido em 11 de fevereiro de 2019

Aceito em 10 de dezembro de 2019

E-mail: karensuzane@hotmail.com 


\section{INTRODUÇÃO}

A utilização de adequados protocolos anestésicos deve levar em consideração, além das demais alterações na fisiologia, as alterações pulmonares causadas pela idade (Mauderly, 1979). Além disso, é fundamental, durante a anestesia, independentemente da idade, o fornecimento de oxigênio, no entanto o seu uso indiscriminado pode acarretar danos pulmonares e celulares, resultando em shunt intrapulmonar (Duggan e Kavanagh, 2005).

Outro cuidado que se deve ter sempre em mente é a depressão respiratória causada pelos anestésicos, elevando os valores de dióxido de carbono, sendo tal achado já evidenciado em estudos anteriores (Fantoni et al., 2016; Bustamante et al., 2018). Nesse cenário, com uma depressão respiratória evidente, devem-se utilizar técnicas adequadas de ventilação.

Entre as modalidades comumente disponíveis e utilizadas em cães, há a ventilação ciclada a volume (VCV) e ciclada a pressão (PCV), podendo-se adicionar nessas a pressão positiva no final da expiração (PEEP). Quando se comparou a VCV e a PCV em cães, não foi verificada diferença entre as modalidades ventilatórias quanto às variáveis hemogasométricas, no entanto foi utilizada a $\mathrm{FiO}_{2}$ de $100 \%$ e PEEP em todas as modalidade, além de serem usados somente animais adultos, entre três e seis anos, podendo tais fatos terem interferido nos resultados (Fantoni et al., 2016). Com isso, não se pode inferir o impacto da modalidade ventilatória nas diferentes faixas etárias encontradas.

Objetivou-se, então, comparar a fração inspirada de oxigênio de $40 \%$ com a fração inspirada de oxigênio de $100 \%$, sob as variáveis cardiovasculares, respiratórias e hemogasométricas, em pacientes jovens, adultos e geriátricos. Além disso, objetivou-se comparar a VCV com volume de ar corrente de $15 \mathrm{~mL} / \mathrm{kg}$ e a PCV com pressão inspiratória de $12 \mathrm{cmH}_{2} \mathrm{O}$ associada ou não à PEEP de $5 \mathrm{cmH}_{2} \mathrm{O}$, em pacientes jovens, adultos e geriátricos.

\section{MATERIAL E MÉTODOS}

Este estudo foi aprovado pelo Comitê de Ética para Uso de Animais (Ceua) da instituição de origem (protocolo de número 9722020317) e conduzido nas dependências do Hospital de
Clínicas Veterinárias da instituição, utilizando-se animais provindos da rotina hospitalar. Foram utilizados 36 cães, sendo 29 fêmeas e sete machos, sem raça definida (SRD), com idade média de $7 \pm 4,3$ anos e peso médio de $16,6 \pm 10,1 \mathrm{~kg}$. Os animais foram anestesiados apenas uma vez, e o procedimento cirúrgico realizado ao final do estudo. Os procedimentos cirúrgicos foram: uma profilaxia dentária, 17 ovário-histerectomias eletivas, sete orquiectomias eletivas, seis mastectomias, quatro nodulectomias e uma vulvoplastia.

Os animais foram submetidos a exames físico e laboratoriais, como hemograma (SDH-3 vet, LabTest) e painel bioquímico (Labmax Plenno, LabTest). Além desses exames, foi realizada também radiografia torácica (HF500M, Lotus-X) e eletrocardiografia (ECGPC Veterinário, TEB). Se algum animal apresentasse quaisquer alterações nos exames citados anteriormente, eles eram encaminhados para o setor clínico do hospital, não sendo alocados no estudo.

O internamento ocorreu no dia anterior ao estudo, sendo os animais submetidos, então, a jejum sólido de 12 horas e hídrico de seis horas. No dia do estudo, foram pré-medicados com morfina $(0,4 \mathrm{mg} / \mathrm{kg})$ e acepromazina $(0,02 \mathrm{mg} / \mathrm{kg})$ pela via intramuscular. Após 15 minutos, foi inserido cateter venoso periférico na veia cefálica e subsequente indução à anestesia geral com propofol dose-efeito. Em ato contínuo, os animais foram intubados com sonda de Murphy de tamanho adequado, conectados ao sistema circular com reinalação parcial de gases e fluxo de gases frescos de $50 \mathrm{~mL} / \mathrm{kg} / \mathrm{min}$. O plano anestésico foi mantido pela vaporização (Tec 7 , Datec-Ohmeda) de isoflurano, mantendo-se a concentração expirada de isoflurano em 1,3V\%, aferida pelo analisador de gases (Módulo ECAIOV-X, GE Carescape B650, Datex-Ohmeda). Durante esse período, os animais permaneciam em ventilação espontânea e com fração inspirada de oxigênio de $100 \%$.

Os pacientes foram, então, posicionados em decúbito dorsal, cateterizando a veia jugular e a artéria femoral com cateter de diâmetro adequado para cada paciente. Com a cateterização, elas foram heparinizadas e conectadas em seus respectivos transdutores de pressão com o zero ajustado no nível do esterno. Com os cateteres inseridos, os pacientes foram, então, alocados em 
dois grupos distintos: grupo 1, que manteve $\mathrm{a} \mathrm{FiO}_{2}$ em $100 \%$; e grupo 0,4 , que teve a $\mathrm{FiO}_{2}$ ajustada para $40 \%$, sendo tal mistura obtida com o auxílio de ar comprimido medicinal. Em ambos os grupos, o fluxo de gases frescos sempre se manteve em $50 \mathrm{~mL} / \mathrm{kg} / \mathrm{min}$, como assinalado anteriormente.

Em cada grupo supracitado, foram alocados $(n=$ 6) animais jovens (GJ), com idade entre um e cinco anos; animais adultos (GA), com idade entre cinco e 10 anos; e animais geriátricos (GG), com idade entre 10 e 15 anos. No grupo 1 e no grupo 0,4 , atentou-se para se alocar o mesmo número de animais $(n=6)$ de cada faixa etária descrita anteriormente.

Após a paramentação e o ajuste da $\mathrm{FiO}_{2}$, atrelada ao grupo ao qual o animal pertencia, eles permaneciam em ventilação espontânea (VE) durante 30 minutos. Após esse período, eram avaliados e submetidos à ventilação ciclada a volume (VCV), com volume de ar corrente de $15 \mathrm{~mL} / \mathrm{kg}$, ou ventilação ciclada a pressão (VCP), com pressão inspiratória de $12 \mathrm{cmH}_{2} \mathrm{O}$, permanecendo 30 minutos em cada modalidade. A ordem entre as modalidades era sorteada em cada animal. A última modalidade empregada era a VCP, com pressão positiva ao final da expiração (VCPP), com pressão inspiratória de $12 \mathrm{cmH}_{2} \mathrm{O}$ e PEEP de $5 \mathrm{cmH}_{2} \mathrm{O}$. As modalidades ventilatórias foram empregadas com o auxílio de um ventilador microprocessado (GE 9100c, Datex-Ohmeda) com relação inspiração:expiração (I:E) 1:2 e frequência respiratória ajustada, a fim de se obter a normocapnia $\left(\mathrm{EtCO}_{2}\right.$ no intervalo $\left.35-45 \mathrm{mmHg}\right)$ (Haskins et al., 2005). Os dados foram coletados após 30 minutos em cada modalidade, totalizando duas horas de estudo. Durante esse período, os animais não foram submetidos à cirurgia, que ocorreu após o término do estudo.

Os parâmetros mensurados durante as avaliações foram obtidos com auxílio do monitor multiparamétrico (GE Carescape B650, DatexOhmeda), sendo esses: frequência cardíaca (FC) em batimentos por minuto; frequência respiratória (f) em movimentos por minuto; pressão arterial sistólica (PAS) em mmHg; pressão arterial média (PAM) em $\mathrm{mmHg}$; pressão arterial diastólica (PAD) em $\mathrm{mmHg}$; concentração expirada de dióxido de carbono $\left(\mathrm{EtCO}_{2}\right)$ em $\mathrm{mmHg}$; pressão de pico (Ppico) em $\mathrm{cmH}_{2} \mathrm{O}$; volume de ar corrente $(\mathrm{Vc})$ em $\mathrm{mL}$; volume minuto $(\mathrm{Vm})$ em $\mathrm{mL} / \mathrm{min}$; complacência pulmonar dinâmica $\left(\mathrm{C}_{\mathrm{T}}\right)$ em $\mathrm{mL} / \mathrm{cm} / \mathrm{H}_{2} \mathrm{O}$; resistência das vias aéreas (RVA) em $\mathrm{cm} / \mathrm{H}_{2} \mathrm{O} / \mathrm{L} / \mathrm{s}$.

Além dos parâmetros mensurados acima, foi realizada também hemogasometria (Cobas b121, Roche) arterial e venosa, mensurando-se: pressão parcial arterial de oxigênio $\left(\mathrm{PaO}_{2}\right)$ em $\mathrm{mmHg}$; pressão parcial arterial de dióxido de carbono $\left(\mathrm{PaCO}_{2}\right)$ em $\mathrm{mmHg}$; potencial de hidrogênio $(\mathrm{pH})$; concentração de bicarbonato $\left(\mathrm{HCO}_{3}\right) \mathrm{em}$ $\mathrm{mmol} / \mathrm{L}$; déficit ou excesso de base (DB ou EB) em mmol/L; saturação arterial de oxigênio $\left(\mathrm{SaO}_{2}\right)$ e saturação venosa de oxigênio $\left(\mathrm{SvO}_{2}\right)$ em \%.

Com esses dados, calculou-se o shunt intrapulmonar (Qs/Qt), utilizando-se a seguinte fórmula: $100 *\left(\mathrm{CcO}_{2}-\mathrm{CaO}_{2}\right) /\left(\mathrm{CcO}_{2}-\mathrm{CvO}_{2}\right)$, em que $\mathrm{CcO}_{2}$ representa o conteúdo capilar de oxigênio, $\mathrm{CaO}_{2}$ representa o conteúdo arterial de oxigênio e $\mathrm{CvO}_{2}$ representa o conteúdo venoso de oxigênio, todos esses dados em mL/dL. $\mathrm{O} \mathrm{CaO}_{2} \mathrm{e}$ o $\mathrm{CvO}_{2}$ são obtidos com a seguinte fórmula: $1,34 * \mathrm{Hb}^{*} \mathrm{SO}_{2}+0,0031 * \mathrm{PO}_{2}$, sendo os valores de $\mathrm{SO}_{2}$ e $\mathrm{PO}_{2}$ os mensurados no sangue arterial e no venoso, obtendo-se, portanto, o $\mathrm{CaO}_{2}$ e o $\mathrm{CvO}_{2}$, respectivamente. $\mathrm{O} \mathrm{CcO}_{2}$ foi obtido utilizando-se a seguinte fórmula: $1,34 * \mathrm{Hb}^{*} \mathrm{SO}_{2}+$ $0,0031 * \mathrm{PAO}_{2}$, em que sempre foi considerada uma saturação capilar de $100 \%$, e a $\mathrm{PAO}_{2}$ foi calculada.

Para o cálculo da $\mathrm{PAO}_{2}$, empregou-se o produto entre $\mathrm{FiO}_{2} *\left(\mathrm{P}_{\mathrm{bar}}-\mathrm{P}_{\text {vapor }}\right)-\mathrm{PaCO}_{2} / \mathrm{RQ}$, em que a Pbar representa a pressão barométrica obtida na hemogasometria, e a Pvapor a pressão de vapor de água $(47 \mathrm{mmHg}$ ). Para o RQ (quociente respiratório), foi utilizado um valor padrão de 0,8 , para pacientes ventilados e recebendo oxigênio suplementar, sendo esse o mesmo utilizado em estudo anterior similar a este (Fantoni et al., 2016). Para o valor da hemoglobina, coletava-se $1 \mathrm{~mL}$ de sangue arterial e venoso, mensurando-se a concentração em contador automático (SDH-3 vet, LabTest). Além disso, calculou-se a relação $\mathrm{PaO}_{2}$ e $\mathrm{FiO}_{2}\left(\mathrm{PaO}_{2} / \mathrm{FiO}_{2}\right)$, sendo essa a relação entre a $\mathrm{PaO}_{2}$ e a $\mathrm{FiO}_{2}$.

Os dados foram submetidos à análise de variância de uma via (One Way - ANOVA), por meio do procedimento PROC MIXED do software estatístico SAS 9.0 (SAS..., 2002), em que se testavam os efeitos individuais da fração inspirada, da idade e da modalidade ventilatória, assim como a interação entre os três. Os dados 
foram, ainda, submetidos à análise de normalidade pelo teste de Shapiro-Wilk; as médias foram testadas pelo teste de Tukey e, quando significativas para interação, elas foram desdobradas $(\mathrm{P} \leq 0,05)$.

\section{RESULTADOS E DISCUSSÃO}

Em relação às idades, os subgrupos apresentaramse diferentes entre as faixas etárias, sem apresentarem diferença entre as frações inspiradas

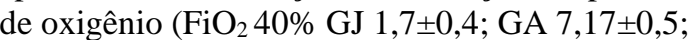
GG $11,8 \pm 0,8$ e $\mathrm{FiO}_{2}$ 100\% GJ 2,9 $\pm 0,7$; GA $6,5 \pm 0,2 ;$ GG 12,5 $\pm 0,5)$. Não houve diferença estatística quanto ao peso dos pacientes $\left(\mathrm{FiO}_{2}\right.$ $40 \%$ GJ 21,9 $\pm 4,45$; GA 9,3 $\pm 1,40$; GG 14,2 $\pm 2,50$ e $\mathrm{FiO}_{2} 100 \%$ GJ 15,6 $\pm 2,58$; GA 21,1 1 7,35; GG $17,3 \pm 3,13)$

Quanto à avaliação dos parâmetros cardiovasculares (Tab. 1), a FC foi maior nos pacientes que receberam a $\mathrm{FiO}_{2}$ de $40 \%$ quando comparada à $\mathrm{FC}$ dos pacientes submetidos à $\mathrm{FiO}_{2}$ de $100 \%$. Esse achado é endossado em estudo anterior, observando-se que elevadas $\mathrm{FiO}_{2}$ reduzem o consumo de oxigênio pelas células, diminuindo, portanto, a FC e o DC em cães conscientes (Lodato, 1989). Com isso, uma possível explicação para essa redução no consumo de oxigênio e consequente redução na FC é a toxicidade do oxigênio, levando paradoxalmente a baixos níveis microcirculatórios dele (Lodato, 1989). Apesar do descrito, os autores não evidenciaram depressão dos valores da FC para valores críticos, não sendo as possíveis alterações na $\mathrm{FC}$ proibitivas à utilização da $\mathrm{FiO}_{2}$ de $100 \%$.

Ainda com relação à FC, esta mostrou-se menor na $\mathrm{VCV}$ quando comparada à $\mathrm{VE}$ e à $\mathrm{VCP}$, sendo na VCPP igual a todas as citadas anteriormente (Tab. 1). Esses achados podem estar atrelados à Ppico, em que, na VCV e na VCPP, foram observados valores de Ppico maiores quando comparados aos valores na VCP (Tab. 2). Essa Ppico maior pode ter induzido a aumento do tônus vagal, levando, por consequência, a uma redução da FC. Tais dados vão de encontro ao descrito na literatura, em que não se observou redução na FC quando comparada a VCV com a VCP (Fantoni et al., 2016). Entretanto, ao empregarem a ventilação mecânica, os autores obtiveram o mesmo valor de Ppico ao empregar-se a VCV e a
VCP, não gerando, portanto, tal diferença entre a FC entre os grupos.

Quanto à FC entre as idades, esse parâmetro foi menor no GJ em relação ao GA e ao GG, fato esse que também ocorreu com relação à PAM. Fisiologicamente, os cães mais jovens, acordados, apresentam FC mais alta em relação aos adultos, devido ao predomínio da atividade simpática. Esse fato, em conjunto com as forças de Franklin Starling não estarem bem desenvolvidas em animais jovens, corroborou a redução da PAM, tendo em vista que, em pacientes jovens, ela é mais dependente da frequência cardíaca. A redução da FC e da PAM apresentada neste estudo pode estar relacionada com a utilização dos fármacos que deprimem o sistema nervoso autônomo simpático, como a morfina (Zickamann et al., 1996).

$\mathrm{Na}$ análise dos parâmetros ventilatórios (Tab. 2), durante a VE a $f$ era regulada pelo paciente, sendo esse valor dependente da ativação das suas respostas compensatórias para as alterações nos valores de $\mathrm{PaCO}_{2}$. Sabe-se que a anestesia deprime, de maneira geral, o bulbo respiratório, portanto, além da redução na $f$, o Vc também pode estar reduzido. Com isso, o Vm irá encontrar-se reduzido, uma vez que essa variável é o produto entre a $f$ e o Vc. Com a redução do $\mathrm{Vm}$, ocorreu o acúmulo de $\mathrm{CO}_{2}$, evidenciado pelos valores maiores de $\mathrm{EtCO}_{2}$ (Tab. 2) e $\mathrm{PaCO}_{2}$ (Tab. 4). Esses achados vão ao encontro dos descritos na literatura (Martins et al., 2003; Fantoni et al., 2016), em que os agentes inalatórios podem deprimir o sistema respiratório.

Na modalidade VCP, a pressão inspiratória é fixada, não sofrendo alterações durante os ciclos respiratórios. As demais variáveis, como fluxo inspiratório e Vc, são dependentes da complacência pulmonar e da resistência das vias aéreas. Com isso, nessa modalidade, se as variáveis de complacência pulmonar ou resistência das vias aéreas sofrerem alterações, o ventilador iria manter a pressão inspiratória igual, já que essa variável é fixada, no entanto o volume corrente entregue iria ser alterado, podendo resultar em acúmulo de $\mathrm{CO}_{2}$ (Consolo et al., 2002; Araújo et al., 2014). 
Efeito de frações...

Tabela 1. Valores médios \pm erro-padrão da frequência cardíaca (FC) em bpm, pressão arterial sistólica (PAS) em mmHg, pressão arterial diastólica (PAD) em mmHg e pressão arterial média (PAM) em mmHg, em cães de três faixas etárias: jovens (0 - 5 anos) (GJ); adultos (5 - 10 anos)(GA); e geriátricos (10 - 15 anos) (GG), submetidos à fração inspirada de oxigênio $\left(\mathrm{FiO}_{2}\right)$ de $40 \%$ ou $100 \%$ e ventilação espontânea (VE) ou mecânica ciclada a volume (VCV), pressão (VCP) ou pressão com PEEP (VCPP), mantidos sob anestesia geral inalatória com $1,3 \mathrm{~V} \%$ de isoflurano

\begin{tabular}{|c|c|c|c|c|}
\hline & $\mathrm{FC}$ & PAS & PAD & PAM \\
\hline \multicolumn{5}{|l|}{$\mathrm{FiO}_{2}(\%)$} \\
\hline 40 & $104 \pm 3 \mathrm{~A}$ & $99 \pm 1$ & $56 \pm 1$ & $69 \pm 1$ \\
\hline 100 & $94 \pm 3 B$ & $96 \pm 1$ & $56 \pm 1$ & $70 \pm 1$ \\
\hline \multicolumn{5}{|l|}{ Modalidade } \\
\hline VE & $105 \pm 4 \mathrm{~A}$ & $94 \pm 2$ & $54 \pm 1$ & $67 \pm 1$ \\
\hline VCV & $89 \pm 4 B$ & $99 \pm 2$ & $56 \pm 1$ & $69 \pm 1$ \\
\hline $\mathrm{VCP}$ & $102 \pm 4 \mathrm{~A}$ & $100 \pm 2$ & $57 \pm 1$ & $71 \pm 1$ \\
\hline VCPP & $97 \pm 4 \mathrm{AB}$ & $98 \pm 2$ & $57 \pm 1$ & $70 \pm 1$ \\
\hline \multicolumn{5}{|l|}{ Idade } \\
\hline GJ & $90 \pm 3 B$ & $95 \pm 2$ & $54 \pm 1$ & $66 \pm 1 \mathrm{~B}$ \\
\hline GA & $102 \pm 3 \mathrm{~A}$ & $100 \pm 2$ & $58 \pm 1$ & $72 \pm 1 \mathrm{~A}$ \\
\hline GG & $103 \pm 3 \mathrm{~A}$ & $97 \pm 2$ & $56 \pm 1$ & $69 \pm 1 \mathrm{AB}$ \\
\hline $\mathrm{P}^{1} \mathrm{FiO}_{2}$ & $0,0216^{*}$ & 0,3376 & 0,6667 & 0,7720 \\
\hline $\mathrm{P}^{1} \mathrm{MOD}$ & $0,0442 *$ & 0,3521 & 0,6944 & 0,4118 \\
\hline $\mathrm{P}^{1} \mathrm{IDADE}$ & $0,0215^{*}$ & 0,2542 & 0,1853 & $0,0206^{*}$ \\
\hline
\end{tabular}

*Valores significativos. Letras maiúsculas na mesma coluna significam diferença estatística entre os grupos (ANOVA), seguida do teste de Tukey $(\mathrm{P} \leq 0,05)$. $\mathrm{P}^{1}$ : probabilidade. MOD - modalidade.

Tabela 2. Valores médios \pm erro-padrão da frequência respiratória $(f)$ em movimentos por minuto, concentração de dióxido de carbono ao final da expiração $\left(\mathrm{EtCO}_{2}\right) \mathrm{em} \mathrm{mmHg}$, volume corrente $(\mathrm{Vc}) \mathrm{em}$

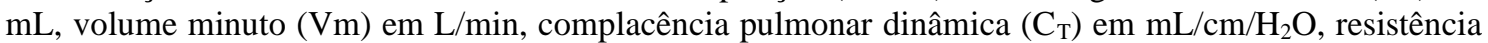
das vias aéreas (RVA) em cm/L/s, e pressão inspiratória de pico (Ppico) em $\mathrm{cmH}_{2} \mathrm{O}$, em cães de três faixas etárias: jovens (0 - 5 anos) (GJ); adultos (5 - 10 anos) (GA); e geriátricos (10 - 15 anos) (GG), submetidos à fração inspirada de oxigênio $\left(\mathrm{FiO}_{2}\right)$ de $40 \%$ ou $100 \%$ e ventilação espontânea (VE) ou mecânica ciclada a volume (VCV), pressão (VCP) ou pressão com PEEP (VCPP), mantidos sob anestesia geral inalatória com $1,3 \mathrm{~V} \%$ de isoflurano

\begin{tabular}{|c|c|c|c|c|c|c|c|}
\hline & $f$ & $\mathrm{EtCO}_{2}$ & $\mathrm{Vc}$ & $\mathrm{Vm}$ & $\mathrm{C}_{\mathrm{T}}$ & RVA & Ppico \\
\hline \multicolumn{8}{|l|}{$\mathrm{FiO}_{2}(\%)$} \\
\hline 40 & $14 \pm 0,8$ & $41 \pm 0,7$ & $216,26 \pm 15,21$ & $3,3 \pm 0,15 \mathrm{~B}$ & $24 \pm 1,8$ & $11 \pm 0,4$ & $10,7 \pm 0,22$ \\
\hline 100 & $16 \pm 0,8$ & $40 \pm 0,7$ & $232,97 \pm 15,21$ & $4,4 \pm 0,15 \mathrm{~A}$ & $26 \pm 1,8$ & $12 \pm 0,4$ & $11,1 \pm 0,22$ \\
\hline \multicolumn{8}{|c|}{ Modalidade } \\
\hline VE & $16 \pm 1,2$ & $45 \pm 1 \mathrm{~A}$ & $200,58 \pm 21,52 \mathrm{~B}$ & $3,2 \pm 0,22 \mathrm{~B}$ & - & - & - \\
\hline VCV & $14 \pm 1,2$ & $36 \pm 1 \mathrm{~B}$ & $271,06 \pm 21,52 \mathrm{~A}$ & $4,4 \pm 0,22 \mathrm{~A}$ & $24 \pm 2,2$ & $13 \pm 0,5 \mathrm{~A}$ & $15,4 \pm 0,31 \mathrm{~A}$ \\
\hline VCP & $16 \pm 1,2$ & $43 \pm 1 \mathrm{~A}$ & $188,25 \pm 21,52 \mathrm{~B}$ & $3,8 \pm 0,22 \mathrm{AB}$ & $25 \pm 2,2$ & $11 \pm 0,5 \mathrm{~B}$ & $10,9 \pm 0,31 \mathrm{~B}$ \\
\hline VCPP & $15 \pm 1,2$ & $38 \pm 1 \mathrm{~B}$ & $238,58 \pm 21,52 \mathrm{AB}$ & $4,0 \pm 0,22 \mathrm{~A}$ & $27 \pm 2,2$ & $10 \pm 0,5 \mathrm{~B}$ & $14,7 \pm 0,31 \mathrm{~A}$ \\
\hline \multicolumn{8}{|l|}{ Idade } \\
\hline GJ & $14 \pm 1 \mathrm{~B}$ & $43 \pm 1 \mathrm{~A}$ & $256,90 \pm 18,63$ & $4,3 \pm 0,19 \mathrm{~A}$ & $32 \pm 2,2 \mathrm{~A}$ & $9 \pm 0,5 \mathrm{~B}$ & $10,9 \pm 0,27$ \\
\hline GA & $15 \pm 1 \mathrm{AB}$ & $38 \pm 1 \mathrm{~B}$ & $217,44 \pm 18,63$ & $4,0 \pm 0,19 \mathrm{~A}$ & $23 \pm 2,2 \mathrm{~B}$ & $12 \pm 0,5 \mathrm{~A}$ & $11,0 \pm 0,27$ \\
\hline GG & $17 \pm 1 \mathrm{~A}$ & $40 \pm 1 \mathrm{~B}$ & $199,52 \pm 18,63$ & $3,2 \pm 0,19 \mathrm{~B}$ & $22 \pm 2,2 \mathrm{~B}$ & $12 \pm 0,5 \mathrm{~A}$ & $10,7 \pm 0,27$ \\
\hline \multicolumn{8}{|l|}{ Anova } \\
\hline $\mathrm{P}^{1} \mathrm{FiO}_{2}$ & 0,0826 & 0,9294 & 0,4390 & $<, 0001 *$ & 0,4439 & 0,1589 & 0,1714 \\
\hline $\mathrm{P}^{1} \mathrm{MOD}$ & 0,6506 & $<, 0001 *$ & $0,0309^{*}$ & $0,0038^{*}$ & 0,6303 & $0,0007^{*}$ & $<, 0001^{*}$ \\
\hline $\mathrm{P}^{1} \mathrm{IDADE}$ & 0,0574 & $0,0009^{*}$ & 0,0880 & $0,0004 *$ & $0,0050^{*}$ & $0,0005^{*}$ & 0,7259 \\
\hline
\end{tabular}

*Valores significativos. Letras maiúsculas na mesma coluna significam diferença estatística entre os grupos (ANOVA), seguida do teste de Tukey $(\mathrm{P} \leq 0,05)$. $\mathrm{P}^{1}$ : probabilidade. MOD - modalidade.

Na VCV, o fluxo é o parâmetro fixado, sendo o volume de ar corrente $(\mathrm{Vc})$ independentemente das demais variáveis, como posição do paciente, alterações na complacência pulmonar e/ou resistência das vias aéreas. Nessa modalidade, o volume corrente foi padronizado em $15 \mathrm{~mL} / \mathrm{kg}$, gerando, com isso, um valor de Ppico maior (Tab. 2) que quando utilizada a VCP, em que se 
padronizou a pressão inspiratória de $12 \mathrm{cmH}_{2} \mathrm{O}$. De maneira análoga à maior Ppico na $\mathrm{VCV}$, o volume corrente, ao empregar-se essa modalidade, foi maior que quando empregada a VCP, uma vez que essa última utilizou uma Ppico menor.

Essas diferenças supracitadas não foram evidenciadas em estudo anterior (Fantoni et al., 2016), em que se comparou a VCV com a VCP em cães anestesiados com isoflurano. Tal achado é creditado à metodologia do estudo, em que os autores padronizaram aumentar a pressão inspiratória na VCP até se obter um valor de volume corrente mensurado pelo monitor de $10 \mathrm{~mL} / \mathrm{kg}$. Já quando empregou a VCV, o volume corrente foi padronizado em $10 \mathrm{~mL} / \mathrm{kg}$ também, ou seja, apesar de as modalidades diferirem quanto à variável fixada, elas foram iguais, uma vez que o volume corrente foi igual em ambas. Em concordância com esses achados, ao se avaliarem valores crescentes de volume correntes em cães com VCV, a pressão inspiratória aumentou de maneira diretamente proporcional ao volume corrente (Oura et al., 2012).

Quanto à VCPP, os valores de $\mathrm{EtCO}_{2}$ e $\mathrm{PaCO}_{2}$ (Tab. 4) permaneceram iguais aos da VCV. Tal fato pode ser creditado ao fato de a Ppico da VCPP ter sido igual à Ppico da VCV, uma vez que, na VCPP, além da pressão inspiratória, terse-ia a PEEP, resultando em uma Ppico maior. Esse achado é reproduzido ao se observar o volume corrente, que foi igual também entre os dois grupos. O emprego de técnicas de PEEP, durante ventilação, diminui o shunt e melhora a oxigenação arterial (Sentürk, 2006). Em estudo conduzido em cães, os pacientes tinham os alvéolos recrutados ou não após meia hora do início da anestesia, permanecendo após a manobra com PEEP de $4 \mathrm{cmH}_{2} \mathrm{O}$ (Cafrán et al., 2012). Essa conduta incrementou a oxigenação nesses pacientes, assim como a complacência (Cafrán et al., 2012).

É valido observar os resultados de outro estudo (De Monte et al., 2013), realizado em cães, que comparou, após a realização do recrutamento alveolar, a utilização da $\mathrm{FiO}_{2}$ de $40 \%$ com a $\mathrm{FiO}_{2}$ de $100 \%$, sendo essa última utilizada sem PEEP ou com PEEP de $5 \mathrm{cmH}_{2} \mathrm{O}$. Os autores encontraram valores iguais de áreas de não aeração (atelectasia) com a $\mathrm{FiO}_{2}$ de $40 \%$ e a FiO2 de $100 \%$ com PEEP, sendo ambos os valores menores que quando utilizada a $\mathrm{FiO}_{2}$ de $100 \%$ sem PEEP. Com isso, é implícito utilizar, então, a PEEP como uma forma de prevenção para o colabamento alveolar, aliado a formas mais prudentes de utilizar o oxigênio, com frações inspiradas mais reduzidas.

A $C_{T}$ foi maior nos animais do $\mathrm{GJ}$ em relação ao GA e ao GG, enquanto a RVA foi maior para o GA e o GG. Muitas são as condições que podem interferir nesses parâmetros, como as fibroses pulmonares, que ocorrem com o envelhecimento, alterando a capacidade de expansão pulmonar, ou a obesidade, que diminui o volume pulmonar e aumenta a resistência, a atelectasia e os shunts pulmonares decorrentes do procedimento anestésico (Lopes e Nunes, 2010; Littleton, 2012). No presente estudo, é possível correlacionar o fato supracitado com a menor área de shunt observada nos animais jovens em comparação aos adultos e geriátricos (Tab. 3). Essas alterações supracitadas são a justificativa para os maiores valores de Vm observados no GJ e no GA.

Com relação aos valores de shunt (Qt/Qs) (Tab. 3), em relação à $\mathrm{FiO}_{2}$, os valores foram maiores na $\mathrm{FiO}_{2}$ de $100 \%$. Esses achados são endossados por estudo anterior, em que, ao empregar-se, após recrutamento alveolar, a $\mathrm{FiO}_{2}$ de $40 \%$, observaram-se menores áreas de atelectasia quando comparada à utilização da $\mathrm{FiO}_{2}$ de $100 \%$ (De Monte et al., 2013). Vale atentar que, neste estudo, não houve diferença entre $\mathrm{a} \mathrm{FiO}_{2}$ de $40 \%$ e a $\mathrm{FiO}_{2}$ de $100 \%$ com PEEP, levando a crer que o aparecimento de atelectasia não é somente devido à elevada $\mathrm{FiO}_{2}$, mas técnicas para a manutenção do alvéolo aberto, como o emprego de PEEP e as manobras de recrutamento, são importantes também. Há algumas justificativas para as maiores áreas de shunt quando instituída a $\mathrm{FiO}_{2}$ de $100 \%$, como a diminuição da produção de surfactante, o que deixa os alvéolos instáveis e com tendência ao colabamento, além da rápida difusão do oxigênio, quando em elevadas concentrações, acarretando o colabamento alveolar (Aboad et al., 2006). 
Efeito de frações...

Tabela 3. Valores médiosterro-padrão de shunt intrapulmonar (Qs/Qt) em porcentagem (\%), relação $\mathrm{PaO}_{2}$ e $\mathrm{FiO}_{2}\left(\mathrm{PaO}_{2} / \mathrm{FiO}_{2}\right)$, saturação arterial de oxigênio $\left(\mathrm{SaO}_{2}\right)$ e saturação venosa de oxigênio $\left(\mathrm{SvO}_{2}\right)$, ambos em \%, em cães de três faixas etárias: jovens (0 - 5 anos) (GJ); adultos (5 - 10 anos) (GA); e geriátricos (10 - 15 anos) (GG), submetidos à fração inspirada de oxigênio $\left(\mathrm{FiO}_{2}\right)$ de $40 \%$ ou $100 \%$ e ventilação espontânea (VE) ou mecânica ciclada a volume (VCV), pressão (VCP) ou pressão com PEEP (VCPP), mantidos sob anestesia geral inalatória com $1,3 \mathrm{~V} \%$ de isoflurano

\begin{tabular}{lllll}
\hline & $\mathrm{Qs} / \mathrm{Qt}$ & $\mathrm{PaO} 2 / \mathrm{FiO} 2$ & $\mathrm{SaO}_{2}$ & $\mathrm{SvO}_{2}$ \\
\hline $\mathrm{FiO}_{2}(\%)$ & & & & \\
40 & $13 \pm 1,4 \mathrm{~B}$ & $491 \pm 7,04 \mathrm{~A}$ & $98 \pm 0,4 \mathrm{~B}$ & $88 \pm 0,6 \mathrm{~B}$ \\
100 & $29 \pm 1,4 \mathrm{~A}$ & $417 \pm 7,04 \mathrm{~B}$ & $99 \pm 0,4 \mathrm{~A}$ & $93 \pm 0,6 \mathrm{~A}$ \\
$\mathrm{MODALIDADE}$ & & & & \\
$\mathrm{VE}$ & $21 \pm 2,0$ & $443 \pm 9,96$ & $99 \pm 0,6$ & $91 \pm 0,8$ \\
$\mathrm{VCV}$ & $20 \pm 2,1$ & $470 \pm 9,96$ & $99 \pm 0,6$ & $89 \pm 0,8$ \\
$\mathrm{VCP}$ & $21 \pm 2,0$ & $451 \pm 9,96$ & $99 \pm 0,6$ & $92 \pm 0,8$ \\
$\mathrm{VCPP}$ & $21 \pm 2,0$ & $452 \pm 9,96$ & $99 \pm 0,6$ & $91 \pm 0,8$ \\
$\mathrm{IDADE}$ & $15 \pm 1,8 \mathrm{~B}$ & $487 \pm 8,62 \mathrm{~A}$ & $99 \pm 0,5$ & $91 \pm 0,7 \mathrm{AB}$ \\
$\mathrm{GJ}$ & $26 \pm 1,8 \mathrm{~A}$ & $453 \pm 8,62 \mathrm{~B}$ & $99 \pm 0,5$ & $92 \pm 0,7 \mathrm{~A}$ \\
$\mathrm{GA}$ & $22 \pm 1,8 \mathrm{~A}$ & $422 \pm 8,62 \mathrm{C}$ & $99 \pm 0,5$ & $89 \pm 0,7 \mathrm{~B}$ \\
$\mathrm{GG}$ & $<, 0001^{*}$ & $<, 0001^{*}$ & & \\
$\mathrm{FiO}$ & 0,9811 & 0,2630 & $<, 0001 *$ & $<, 0001 *$ \\
$\mathrm{Modalidade}^{*}$ & $0,0005^{*}$ & $<, 0001^{*}$ & 0,3557 & 0,1502 \\
$\mathrm{Idade}$ & $0,0291^{*}$ & $0,0009^{*}$ & 0,3295 & $0,0038^{*}$ \\
$\mathrm{FiO}_{2}$ X Idade & & & & \\
\hline
\end{tabular}

*Valores significativos. Letras maiúsculas na mesma coluna significam diferença estatística entre os grupos (ANOVA), seguida do teste de Tukey $(\mathrm{P} \leq 0,05) . \mathrm{P}^{1}$ : probabilidade. $\mathrm{MOD}$ - modalidade.

Quanto à relação $\mathrm{PaO}_{2}$ e $\mathrm{FiO}_{2}\left(\mathrm{PaO}_{2} / \mathrm{FiO}_{2}\right)(\mathrm{Tab}$. 3 ), os valores foram significativamente maiores para a $\mathrm{FiO}_{2}$ de $40 \%$ e diferiram entre as idades, sendo encontrados valores maiores no GJ seguido do GA e do GG. A relação $\mathrm{PaO}_{2}$ e $\mathrm{FiO}_{2}$ é calculada pela razão entre $\mathrm{PaO}_{2} / \mathrm{FiO}_{2}$, que reflete a adequada ventilação pulmonar $\mathrm{e}$ as trocas gasosas. Essa diferença é justificada pelos valores de shunt maiores conforme maior a $\mathrm{FiO}_{2}$ e a idade, afetando, dessa forma, a troca gasosa e a oxigenação. Tal achado vai ao encontro do descrito por De Monte et al. (2013), os quais evidenciaram também menores valores da relação $\mathrm{PaO}_{2} / \mathrm{FiO}_{2}$ após recrutamento alveolar e manutenção com a $\mathrm{FiO}_{2}$ de $100 \%$ quando comparada à $\mathrm{FiO}_{2}$ de $40 \%$. Com isso, este estudo reforça o pressuposto de que $\mathrm{FiO}_{2}$ menores são mais adequadas para a ocorrência da troca gasosa.

Para a $\mathrm{SaO}_{2}$ (Tab. 3), todas as médias foram maiores que 95\%, com o emprego das duas $\mathrm{FiO}_{2}$. Já para a $\mathrm{SvO}_{2}$, houve diferença em relação às $\mathrm{FiO}_{2}$ e às idades, sendo os valores menores encontrados na $\mathrm{FiO}_{2}$ de $40 \%$ e no GG em relação ao GJ e ao GA. Os valores considerados fisiológicos situam-se entre $65 \%$ e $75 \%$ (Silva et al., 2001), sendo observados valores maiores que

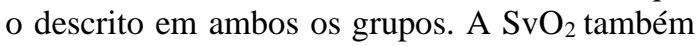

pode ser influenciada por diferentes valores de $\mathrm{FiO}_{2}$, uma vez que a $\mathrm{SvO}_{2}$ é o balanço entre o consumo e a oferta de oxigênio (Haskins et al., 2005), ou seja, ao se incrementar a $\mathrm{FiO}_{2}$, pode-se incrementar o conteúdo arterial de oxigênio e, consequentemente, a oferta irá aumentar também proporcionalmente. Valores maiores que $75 \%$ são obtidos em estados de baixo consumo de $\mathrm{O}_{2}$, como o que acontece nas condições de anestesia e bloqueio neuromuscular, fato esse que ocorreu neste estudo.

Com relação às diferenças encontradas quanto à $\mathrm{PaO}_{2}$ (Tab. 4), com a $\mathrm{FiO}_{2}$ de $100 \%$ esse valor foi maior em relação à $\mathrm{FiO}_{2} 40 \%$. $\mathrm{O}$ valor de $\mathrm{PaO}_{2}$ irá aumentar com o aumento da $\mathrm{FiO}_{2}$, entretanto, mesmo com um valor de $\mathrm{PaO}_{2}$ elevado, esse isolado não irá indicar uma oxigenação adequada, devendo-se sempre relacionar a $\mathrm{PaO}_{2}$ com a FiO 2 , sendo uma dessas formas a razão entre $\mathrm{PaO}_{2} \mathrm{e}$ $\mathrm{FiO}_{2} \quad\left(\mathrm{PaO}_{2} / \mathrm{FiO}_{2}\right)$, discutida anteriormente. Quando analisada conjuntamente com a $\mathrm{PaCO}_{2}$, nota-se que esta apresentou-se maior ao empregar-se $\mathrm{a} \mathrm{FiO}_{2}$ de $100 \%$ quando comparada à $\mathrm{FiO}_{2}$ de $40 \%$. Esses achados podem ser creditados aos valores elevados de shunt intrapulmonar na $\mathrm{FiO}_{2}$ de $100 \%$, o que sugere que altas $\mathrm{FiO}_{2}$ aumentam os valores de shunt e, assim, dificultam 
as trocas gasosas, resultado que corrobora o observado por outros autores que utilizaram diferentes $\mathrm{FiO}_{2}$ durante procedimentos anestésicos em cães (Lopes et al., 2007; De Monte et al., 2013).

Os valores de $\mathrm{PaO}_{2}$ foram maiores no GJ, decrescendo com o avanço da faixa etária, sendo encontrandos os menores valores no GG. Os cães atingem o ápice da capacidade pulmonar com um ano de idade, iniciando-se, após essa idade, o processo de envelhecimento, o que reduz a eficiência nas trocas gasosas, principalmente pela redução na complacência pulmonar (Mauderly 1979). A influência desse processo de envelhecimento interferiu diretamente nos valores de Vc, Vm, $\mathrm{C}_{\mathrm{T}}, \mathrm{PaO}_{2}$, Qs/Qt e $\mathrm{PaO}_{2} / \mathrm{FiO}_{2}$, que se reduziram com a idade, além de a RVA ter aumentado com a idade.

Os valores de $\mathrm{PaCO}_{2}$ (Tab. 4) foram maiores ao empregar-se a $\mathrm{FiO}_{2}$ de $100 \%$. Entre as modalidades, essa variável foi maior ao utilizar a VE e a VCP, estando fora do intervalo aceitável para espécie (Haskins et al., 2005). Esse aumento nos valores de $\mathrm{PaCO}_{2}$ em cães já foi relacionado a elevados valores de $\mathrm{FiO}_{2}$, como de $80 \%$ e $100 \%$, sendo esses resultados atrelados a um aumento nas áreas de atelectasia e shunt (Lopes et al., 2007; Nunes et al., 2008). Tal fato foi evidenciado também no presente estudo.

Ao se relacionarem os valores de $\mathrm{PaCO}_{2}$ com a modalidade ventilatória, esses achados foram atrelados à depressão respiratória causada pelos anestésicos de maneira geral, ao se utilizar a VE, ao passo que, ao se utilizar a VCP, o Vm foi inferior à VCV e à VCPP; dessa forma, houve redução na ventilação alveolar e acúmulo de $\mathrm{CO}_{2}$. Esses achados vão ao encontro da literatura (Fantoni et al., 2016), em que também foram observados valores elevados de $\mathrm{PaCO}_{2}$ $(54 \mathrm{mmHg})$ durante a VE.

O pH arterial (Tab. 4) apresentou diferença significativa entre as modalidades ventilatórias, observando-se valores menores na VE e na VCP em relação à VCV e à VCPP. Essas diferenças são justificáveis pelas alterações encontradas nos valores de $\mathrm{PaCO}_{2}$, cursando com uma leve acidemia de origem respiratória, sendo a causa disso possivelmente o Vm menor ao se utilizar a VCP, aliado à depressão respiratória provocada pela anestesia. Em relação ao DB (Tab. 4), a diferença estatística se deu para as diferentes $\mathrm{FiO}_{2}$, sendo valores menores atribuídos para a $\mathrm{FiO}_{2} 40 \%$. Essa variável demonstra o efeito compensatório da acidose respiratória, por meio do consumo das bases disponíveis ao tamponamento do $\mathrm{pH}$.

Com relação às limitações encontradas no estudo e que podem ter influenciado os resultados, uma delas foi a não padronização na escolha do procedimento. Apesar de tal limitação, os autores acreditam que isso não influenciou negativamente o resultado, uma vez que os animais sempre realizavam radiografias torácicas, a fim de se excluírem quaisquer causas de comprometimento pulmonar preexistente. Além disso, ao empregarem-se diferentes situações cirúrgicas, foram obtidad diferentes condições, o que normalmente ocorre no âmbito clínico. Dessa forma, os resultados aqui encontrados são aplicáveis à rotina clínica, visto que as condições não foram todas padronizadas.

Outra limitação do estudo foi a padronização do volume corrente e da pressão de pico, fazendo, dessa forma, com que os resultados aqui encontrados sejam atribuídos aos valores utilizados no estudo. Entretanto, os autores partiram desses valores devido à sua utilização nas anestesias do hospital de origem. Além disso, cabe ressaltar que, no presente estudo, avaliaramse três fatores $\left(\mathrm{FiO}_{2}\right.$, modo ventilatório e idade), sendo, portanto, dificultoso incluir mais um fator para gerar variação entre os grupos. Com isso, é implícito que se devem realizar estudos futuros utilizando as $\mathrm{FiO}_{2}$ reduzidas e avaliando isoladamente diferentes pressões de pico e diferentes volumes de ar corrente. 
Efeito de frações...

Tabela 4. Valores médios terro-padrão da pressão parcial arterial de oxigênio $\left(\mathrm{PaO}_{2}\right)$ em $\mathrm{mmHg}$, pressão parcial arterial de gás carbônico $\left(\mathrm{PaCO}_{2}\right) \mathrm{em} \mathrm{mmHg}$, potencial de hidrogênio $(\mathrm{pH})$, bicarbonato $\left(\mathrm{HCO}_{3}\right) \mathrm{em}$ $\mathrm{mmol} / \mathrm{L}$ e déficit de base (DB) em mmol/L, em cães de três faixas etárias: jovens ( 0 - 5 anos) (GJ); adultos (5 - 10 anos) (GA); e geriátricos (10 - 15 anos) (GG), submetidos à fração inspirada de oxigênio $\left(\mathrm{FiO}_{2}\right)$ de $40 \%$ ou $100 \%$ e ventilação espontânea (VE) ou mecânica ciclada a volume (VCV), pressão (VCP) ou pressão com PEEP (VCPP), mantidos sob anestesia geral inalatória com 1,3V\% de isoflurano.

\begin{tabular}{|c|c|c|c|c|c|}
\hline & $\mathrm{PaO}_{2}$ & $\mathrm{PaCO}_{2}$ & $\mathrm{pH}$ & $\mathrm{HCO}_{3}$ & DB \\
\hline \multicolumn{6}{|l|}{$\mathrm{FiO}_{2}(\%)$} \\
\hline 40 & $196,4 \pm 5,26 \mathrm{~B}$ & $43,1 \pm 0,85$ B & $7,30 \pm 0,005$ & $20,34 \pm 0,264 \mathrm{~B}$ & $-5,5 \pm 0,16 \mathrm{~B}$ \\
\hline 100 & $417,9 \pm 5,26 \mathrm{~A}$ & $45,9 \pm 0,85 \mathrm{~A}$ & $7,31 \pm 0,005$ & $22,78 \pm 0,264 \mathrm{~A}$ & $-3,9 \pm 0,16 \mathrm{~A}$ \\
\hline \multicolumn{6}{|l|}{ Modalidade } \\
\hline VE & $298,2 \pm 7,44$ & $49,1 \pm 1,21 \mathrm{~A}$ & $7,27 \pm 0,007 \mathrm{C}$ & $21,93 \pm 0,373$ & $-4,8 \pm 0,22$ \\
\hline VCV & $320,7 \pm 7,44$ & $39,0 \pm 1,21 \mathrm{~B}$ & $7,34 \pm 0,007 \mathrm{~A}$ & $21,09 \pm 0,373$ & $-4,6 \pm 0,22$ \\
\hline VCP & $306,6 \pm 7,44$ & $47,7 \pm 1,21 \mathrm{~A}$ & $7,29 \pm 0,007 \mathrm{C}$ & $21,95 \pm 0,373$ & $-4,7 \pm 0,22$ \\
\hline VCPP & $303,1 \pm 7,44$ & $42,2 \pm 1,21 \mathrm{~B}$ & $7,31 \pm 0,007 \mathrm{~B}$ & $21,27 \pm 0,373$ & $-4,8 \pm 0,22$ \\
\hline \multicolumn{6}{|l|}{ Idade } \\
\hline GJ & $332,3 \pm 6,45 \mathrm{~A}$ & $43,2 \pm 1,04$ & $7,31 \pm 0,006$ & $21,01 \pm 0,323$ & $-5,0 \pm 0,19$ \\
\hline GA & $312,1 \pm 6,45$ B & $44,0 \pm 1,04$ & $7,30 \pm 0,006$ & $21,82 \pm 0,323$ & $-4,7 \pm 0,19$ \\
\hline GG & $277,2 \pm 6,45 \mathrm{C}$ & $46,4 \pm 1,04$ & $7,30 \pm 0,006$ & $21,85 \pm 0,323$ & $-4,4 \pm 0,19$ \\
\hline $\mathrm{P}^{1} \mathrm{FiO}_{2}$ & $<, 0001^{*}$ & $0,0215^{*}$ & 0,1844 & $<, 0001^{*}$ & $<, 0001^{*}$ \\
\hline $\mathrm{P}^{1}$ Modalidade & 0,1734 & $<, 0001 *$ & $<, 0001 *$ & 0,2428 & 0,9395 \\
\hline $\mathrm{P}^{1}$ Idade & $<, 0001 *$ & 0,0863 & 0,7738 & 0,1153 & 0,1431 \\
\hline
\end{tabular}

*Valores significativos. Letras maiúsculas na mesma coluna significam diferença estatística entre os grupos (ANOVA), seguida do teste de Tukey $(\mathrm{P} \leq 0,05)$. $\mathrm{P}^{1}$ : probabilidade. MOD - modalidade.

\section{CONCLUSÕES}

Conclui-se que a fração inspirada de $40 \%$ e a ventilação ciclada a volume, com volume corrente de $15 \mathrm{ml} / \mathrm{kg}$, ou ciclada a pressão, com pressão inspiratório de $12 \mathrm{cmH} 2 \mathrm{O}$, associada a pressão positiva ao final da expiração (PEEP) de $5 \mathrm{cmH}_{2} \mathrm{O}$, manteve os parâmetros ventilatórios mais adequados quando comparada a ciclagem a pressão sem PEEP. Os resultados estão atrelados ao volume e pressão utilizados no estudo, devendo-se individualizar cada um destes parâmetros em cada animal de diferentes faixas etárias.

\section{REFERÊNCIAS}

ABOAD, J.; JONSON, B.; KOUATCHET, A. et al. Effect of inspired oxygen fraction on alveolar derecruitment in acute respiratory distress syndrome. Intensiv. Care Med., v.32, p.19791986, 2006.

ARAÚJO, D.A.; LEÃO, B.C.C.; FERREIRA, R.S. Volume x pressão. Qual escolher? Rev. Méd. Minas Gerais, v.24, p.49-55, 2014.

BUSTAMANTE, R.; AGUADO, D.; CEDIEL, R. et al. Clinical comparison of the effects of isoflurane or propofol anaesthesia on mean arterial blood pressure and ventilation in dogs urdergoing orthopaedic surgery receiving epidural anaesthesia. Vet. J., v.223, p.49-54, 2018.
CANFRÁN, S.; GÓMEZ DE SEGURA, I. A.; CEDIEL, R.; et al. Effects of a stepwise lung recruitment manoeuvre and positive endexpiratory pressure on lung compliance and arterial blood oxygenation in healthy dogs. Vet. J., v. 194, p. 89-93, 2012.

CONSOLO, L.C.T.; PALHARES, D.B.; CONSOLO, L.Z.Z. Avaliação da função pulmonar de recém-nascidos com síndrome do desconforto respiratório em diferentes pressões finais expiratórias positivas. J. Pediatr., v.78, p.403-408, 2002.

DE MONTE, V.; GRASSO, S.; DE MARZO, C. et al. Effects of the reduction of inspired oxygen fraction or application of positive end-expiratory pressure after an alveolar recruitment maneuver on respiratory mechanics, gas exchange, and lung aeration in dogs during anesthesia and neuromuscular blockade. Am. J. Vet. Res., v.74, p.25-33, 2013.

DUGGAN, M.; KAVANAGH, B.P. Pulmonary atelectasis: a pathogenic perioperative entity. Anesthesiology, v.102, p.838-854, 2005.

FANTONI, D.T.; IDA, K.K.; LOPES, T.F.T. et al. A comparison of the cardiopulmonary effects of pressure controlled ventilation and volume controlled ventilation in healthy anesthetized dogs. J. Vet. Emerg. Crit. Care, v.26, p.524-530, 2016. 
HASKINS, S.; PASCOE, P.J.; ILKIW, J.E. et al. Reference cardiopulmonary values in normal dogs. Comp. Med., v.55, p.156-161, 2005.

LITTLETON, S.W. Impact of obesity on respiratory function. Respirology, v.17, p.43-49, 2012.

LODATO, R.F. Decreased oxygen consumption and cardiac output during normobaric hiperoxia in conscious dogs. J. Appl. Physiol., v.67, p.15511559, 1989.

LOPES, P.C.F.; NUNES, N. Atelectasia pulmonar em cães durante anestesia geral. Cienc. Rural, v.40, p.246-253, 2010.

LOPES, P.C.F.; NUNES, N.; CARARETO, R. et al. Efeitos de diferentes frações inspiradas de oxigênio sobre a dinâmica respiratória em cães submetidos à infusão contínua de propofol e mantidos em ventilação espontânea. Braz. J. Vet. Res. Anim. Sci., v.44, p.30-37, 2007.

MARTINS, S.E.C.; NUNES, N.; REZENDE, M.L. et al. Efeitos do desflurano, sevoflurano e isoflurano sobre variáveis respiratórias e hemogasométricas em cães. Braz. J. Vet. Res. Anim. Sci., v.40, p.190-196, 2003.
MAUDERLY, J.L. Effect of age on pulmonary structure and function of immature and adult animals and man. Fed. Proc., v.138, p.173-177, 1979

NUNES, N.; LOPES, P.C.F.; SANTOS, P.S.P. et al. Hemodinâmica de diferentes frações inspiradas de oxigênio em cães submetidos à infusão contínua de propofol sob ventilação espontânea. Cienc. Rural, v.38, p.729-735, 2008.

OURA, T.; ROZANSKI, E.A.; BUCKLEY, G. et al. Low tidal volumen ventilation in healthy dogs. J. Vet. Emerg. Crit. Care, v.22, p.368-371, 2012.

SENTÜRK, M. New concepts of management of one-lung ventilation. Curr. Opin Anaesthesio., v.19, p.1-4, 2006.

SILVA, E.; GARRIDO, G.A.; ASSUNÇÃO, M.S.C. Avaliação da perfusão tecidual no choque. Medicina., v.34, p.27-35, 2001.

ZICKMANN, B.; HOFMANN, H.C.; POTTKAMPER, C. et al. Changes in heart rate variability during induction of anesthesia with fentanyl and midazolam. J. Cardiothor. Vasc. An., v.10, p.609-613, 1996 